\title{
Pixel Level Smoke Detection Model with Deep Neural Network
}

Muthukumaran Ramasubramanian' ', Aaron Kaulfus' ', Manil Maskey², Rahul

Ramachandran'2, Iksha Gurung' ', Brian Freitag' ', Sundar Christopher'

1-University of Alabama in Huntsville

2-NASA/Marshall Space Flight Center 


\section{Introduction}

- Biomass burning smoke has numerous detrimental environmental and ecological impacts

- Respiratory and cardiovascular illnesses

- Radiation budget

- Nutrient availability

- Impacts realized both near source and potentially thousands of kilometers downwind depending on

- Fire duration

- Amount and type of biomass burned

- Meteorological and fuel conditions

- Vertical distribution in the atmosphere
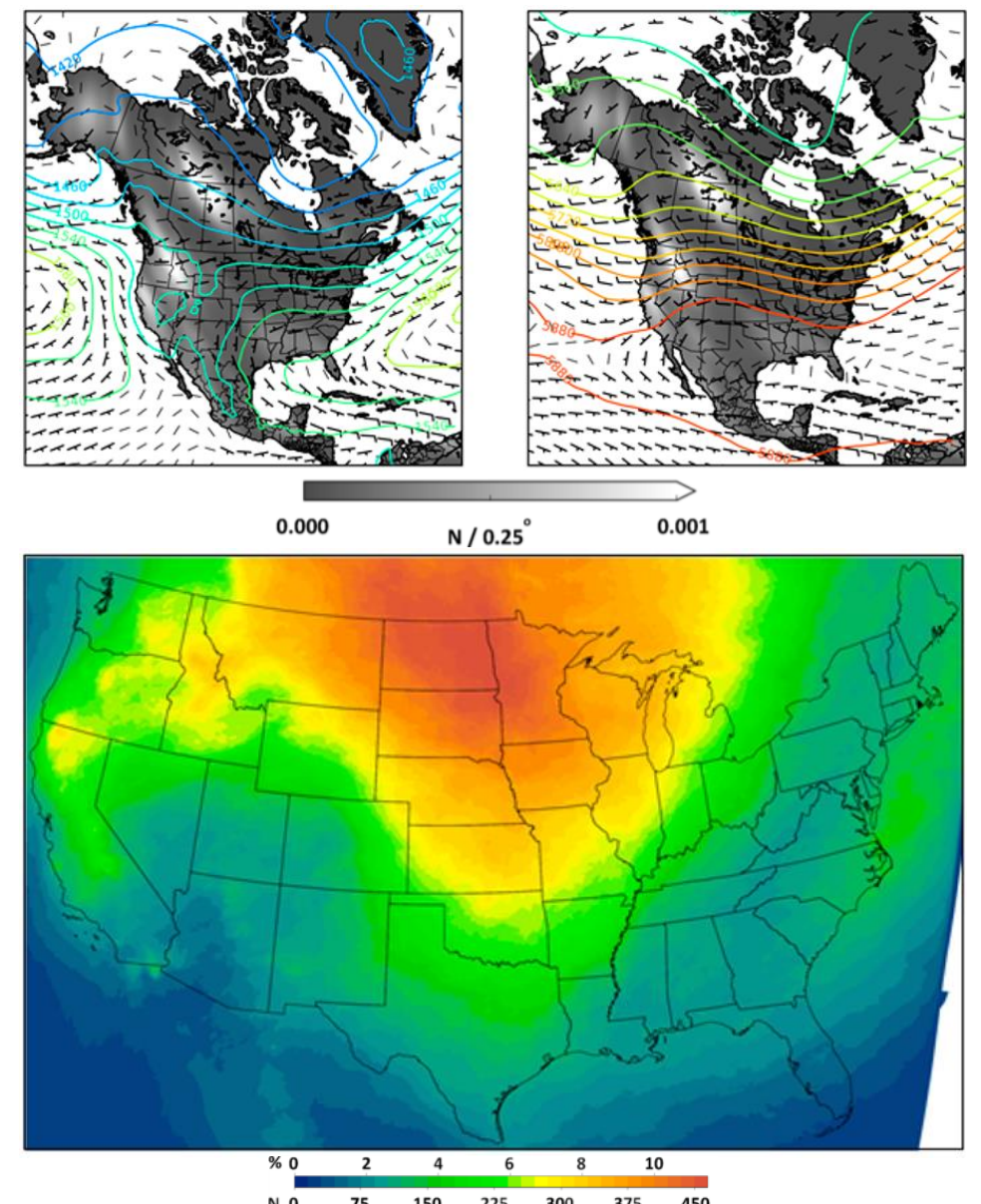

Spatial distribution of MDDIS fire accurrence and NDAA HMS smoke for summer 2006-2015. Fram Kaulfus et al. 2017 Figure 2. 


\section{Introduction}

- Current methods present challenges for continuous smoke detection and monitoring

- In-situ monitoring

- Temporal, spatial, and tracer limitations

- Remote sensing

- Polar orbiting, once-daily overpass

- Manual or computational intensive multispectral analysis

- Large data volumes

- Multiple class multispectral classification 


\section{Objectives}

- Deploy a smoke detection model using machine learning on satellite remote sensing observations

- Leverage observations from the new generation of geostationary satellite

- High spatial and temporal resolutions over large domains

- Alternative to multispectral analysis

- Eliminate time consuming, subjective manual analysis 


\section{Truth Dataset}

- Geostationary Operational Environmental Satellite 16 shortwave reflectance data

- Bands 1-6 (0.47, 0.64, 0.86, 1.37, 1.6 and $2.2 \mu \mathrm{m})$

- Access L1B radiance data from AWS

- Convert to reflectance

- Spatially resample to $1 \mathrm{~km}$

- National Oceanic and Atmospheric Administration (NOAA) Hazard Mapping System (HMS) smoke analysis

- Satellite based operational daily analysis of smoke extent over the US and surrounding areas

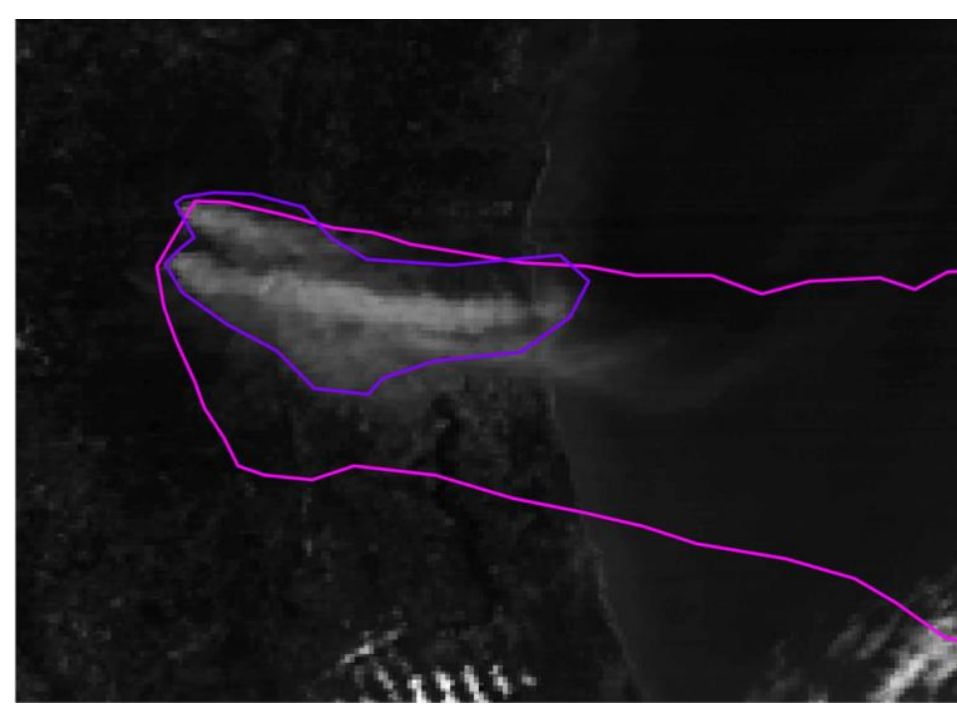

GOES 16 band 1 radiance with nearest in time HMS shapefiles (magenta and purple)

- Manual quality controlled by subject matter expert to correctly match smoke extent in GOES 16 image 


\section{Truth Dataset}

- Geostationary Operational Environmental Satellite 16 shortwave reflectance data

- Bands 1-6 (0.47, 0.64, 0.86, 1.37, 1.6 and $2.2 \mu \mathrm{m})$

- Access L1B radiance data from AWS

- Convert to reflectance

- Spatially resample to $1 \mathrm{~km}$

- National Oceanic and Atmospheric Administration (NOAA) Hazard Mapping System (HMS) smoke analysis

- Satellite based operational daily analysis of smoke extent over the US and surrounding areas

- Manual quality controlled by subject matter expert

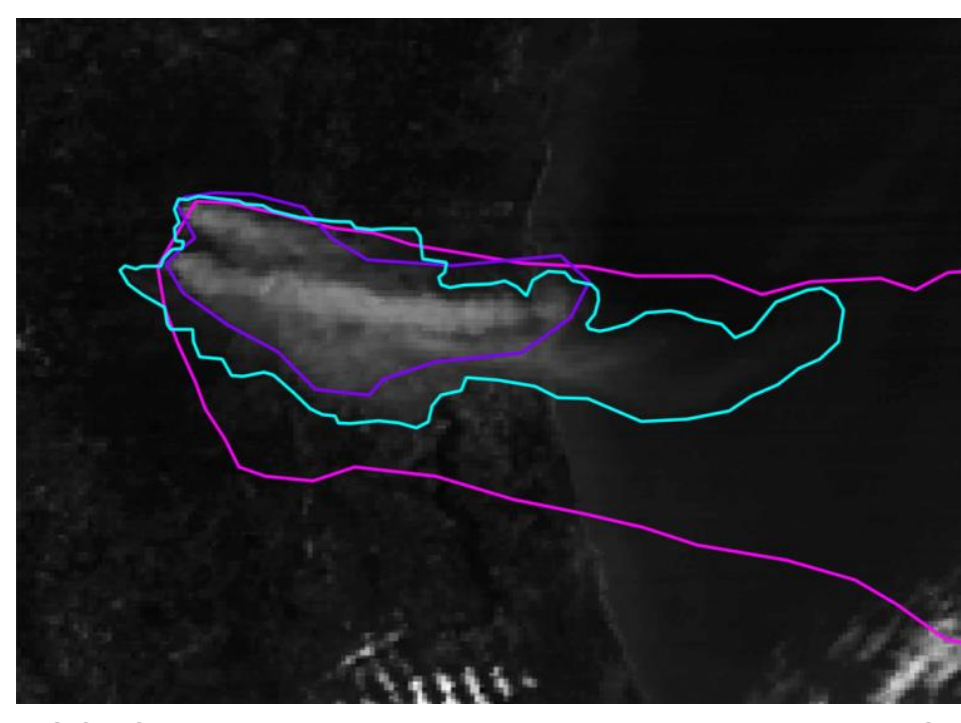

GOES 16 band 1 radiance with nearest in time HMS shapefiles (magenta and purple) with subject matter quality controlled shapefile (blue). to correctly match smoke extent in GOES 16 image 


\section{Truth Dataset}

- Analyze 122 scenes containing smoke

- 962,691 smoke pixels

- Over low and high background reflectances (land and ocean)

- Low and high optical thicknesses

- Full range of sun angles

- Contain relevant classes to discriminate smoke from including

- Snow and ice

- Clouds

- Dust

- $60 \%-20 \%-20 \%$ distribution of smoke pixels between training, validation and testing datasets 


\section{Model Architecture}

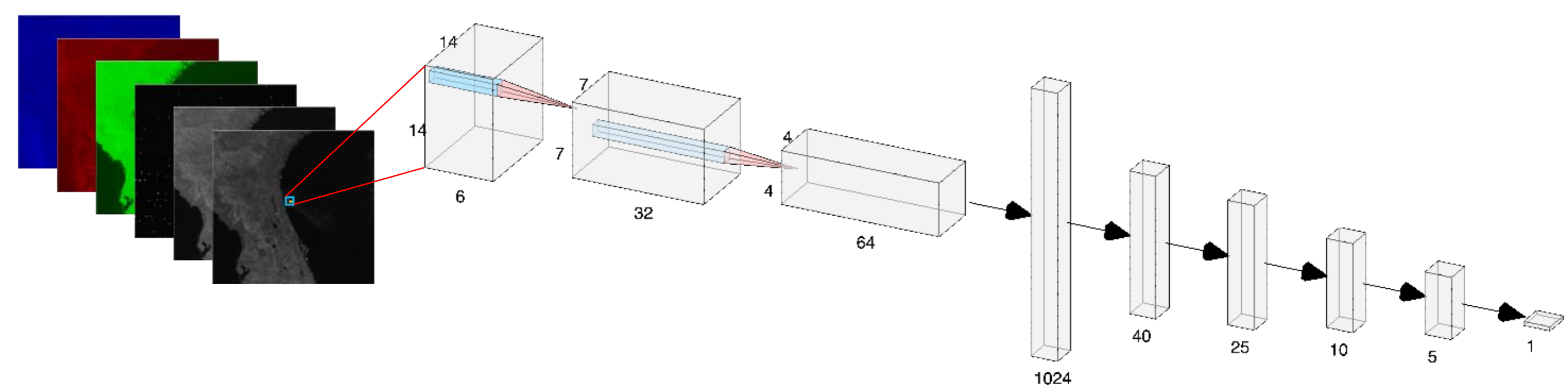

- Apply a pixel based Convolutional Neural Network (CNN)

- Input $\left(N^{*} 2\right)^{*}\left(N^{*} 2\right)$ neighborhood of reflectance values surrounding a center pixel (sample)

- 3 convolutional layers

- Each convolutional layer followed by max-pooling layer

- Convolutional outputs are flattened into vectors 


\section{Model Architecture}
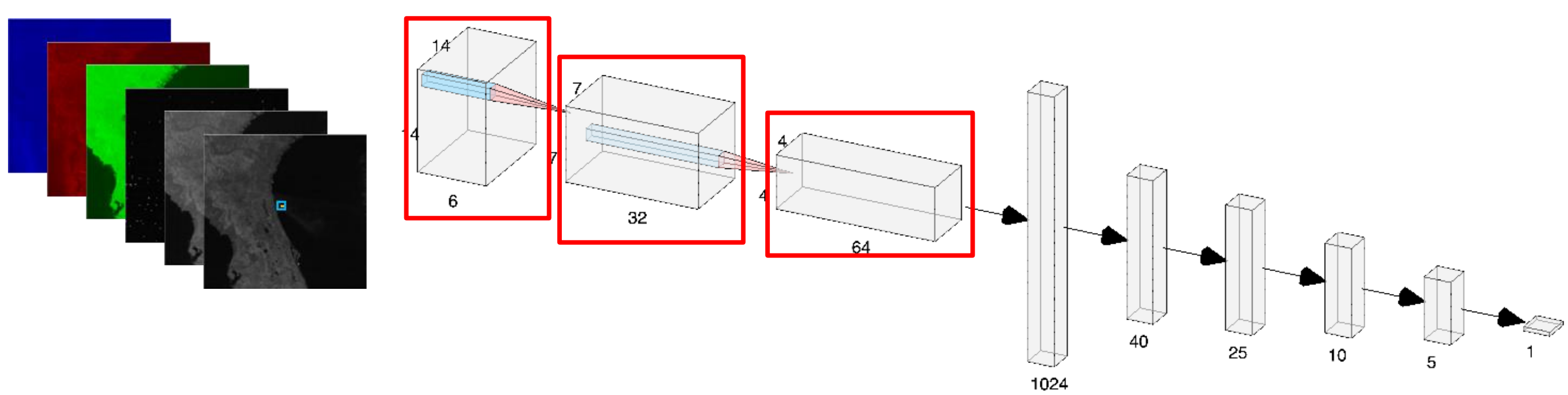

- Apply a pixel based Convolutional Neural Network (CNN)

- Input $\left(\mathrm{N}^{\star} 2\right)^{\star}\left(\mathrm{N}^{\star} 2\right)$ neighborhood of reflectance values surrounding a center pixel (sample)

- 3 convolutional layers

- Each convolutional layer followed by max-pooling layer

- Convolutional outputs are flattened into vectors 


\section{Model Architecture}
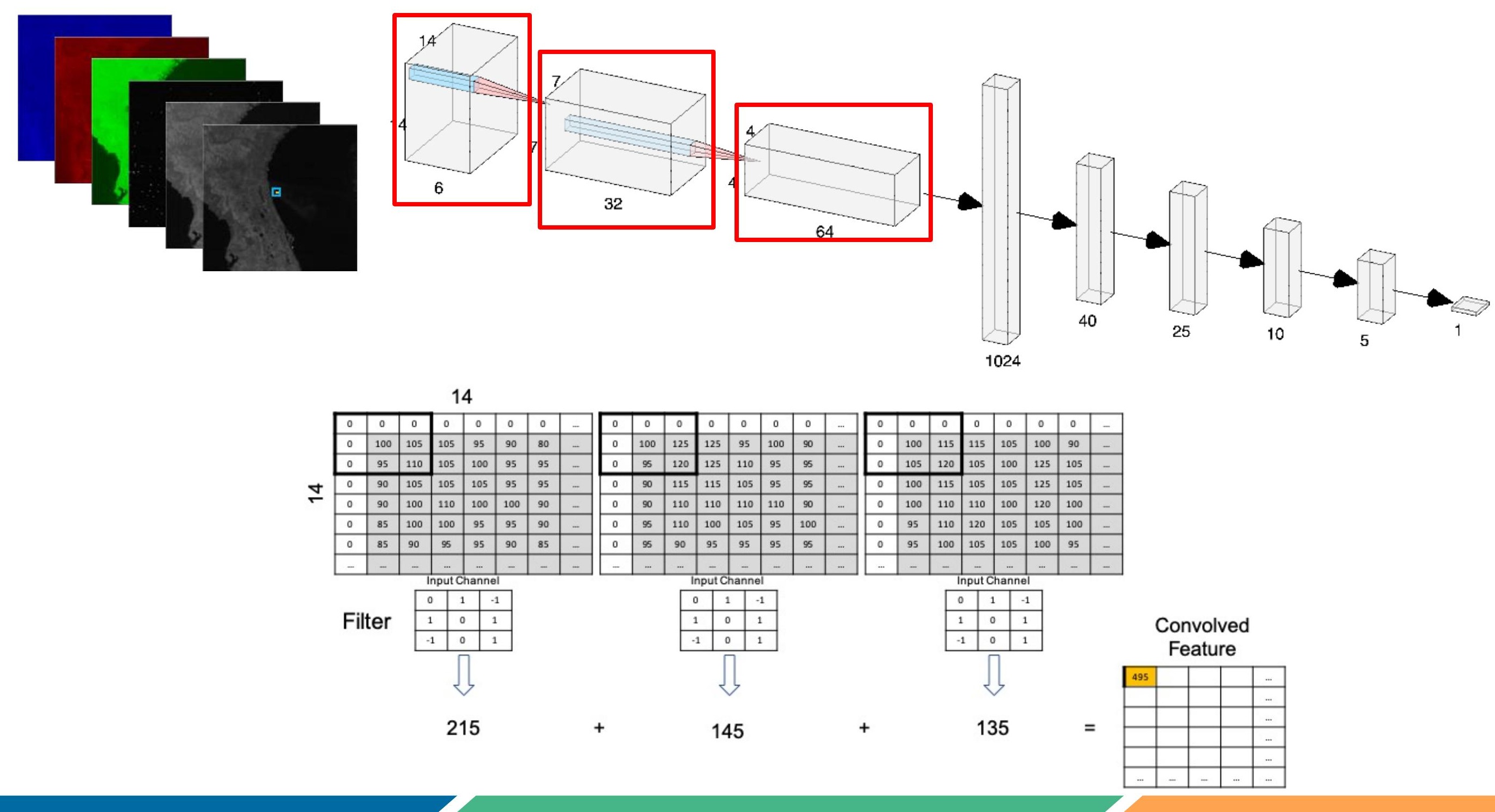


\section{Model Architecture}
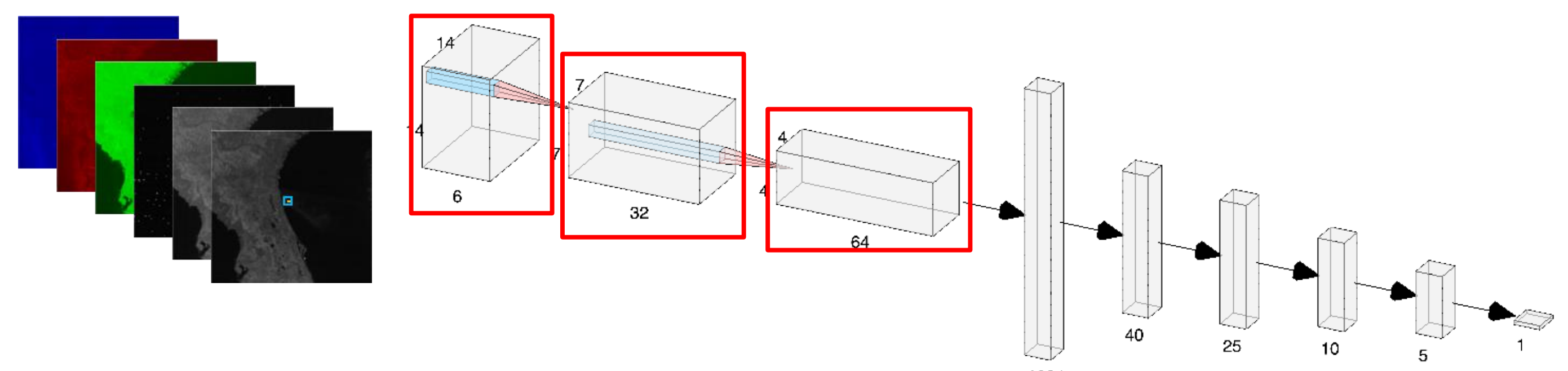

14
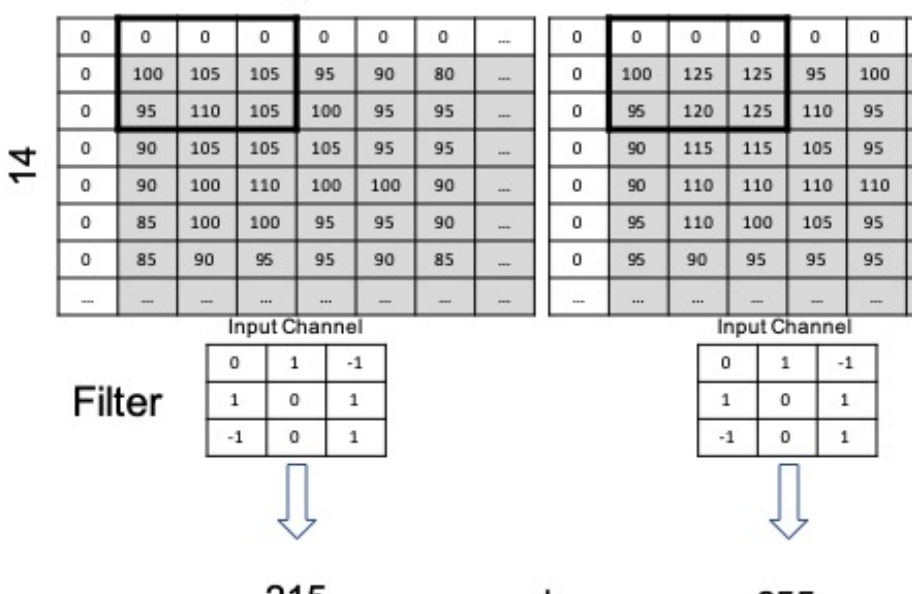

255
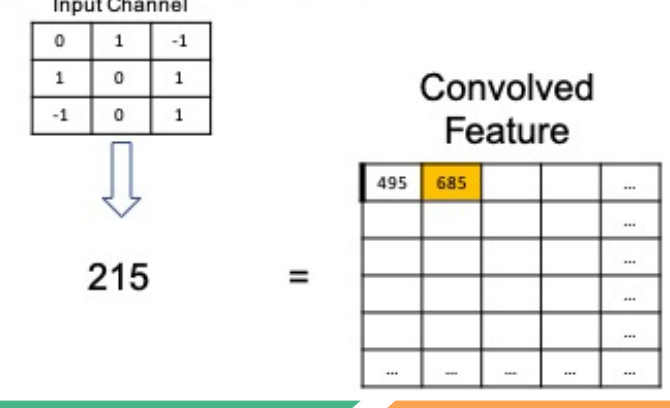


\section{Model Architecture}
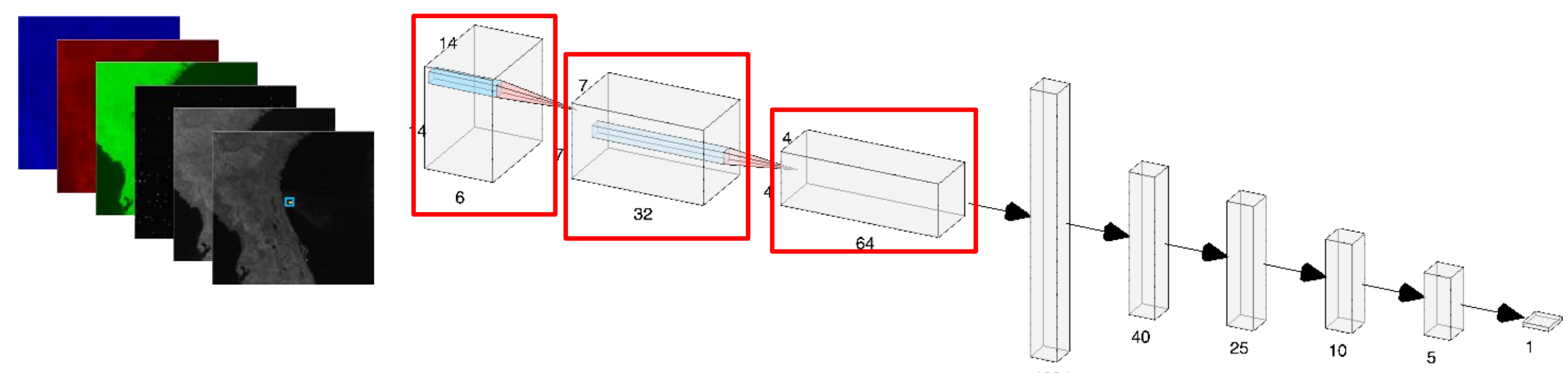

14

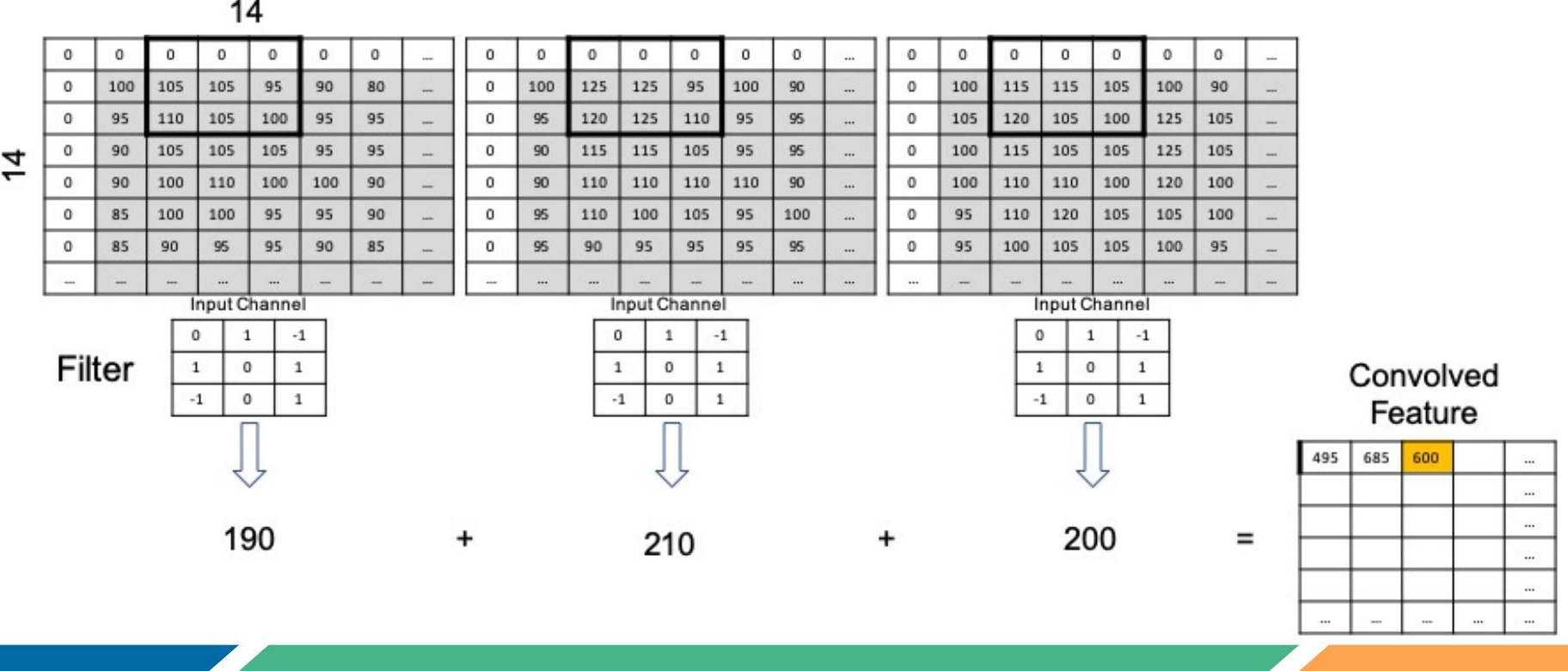




\section{Model Architecture}
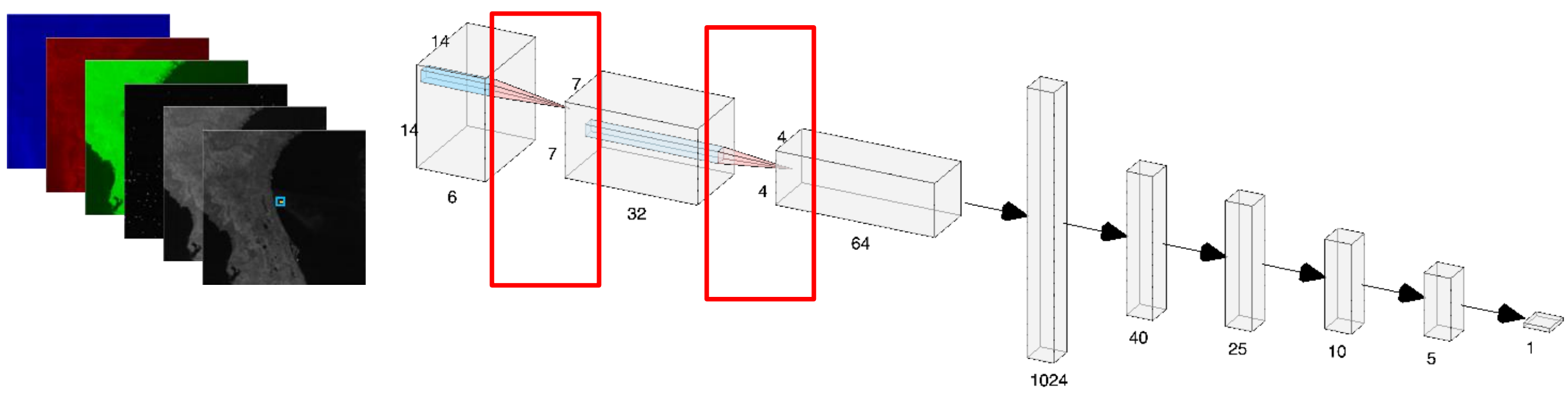

- Apply a pixel based Convolutional Neural Network (CNN)

- Input $\left(\mathrm{N}^{\star} 2\right)^{\star}\left(\mathrm{N}^{\star} 2\right)$ neighborhood of reflectance values surrounding a center pixel (sample)

- 3 convolutional layers

- Each followed by max-pooling layer

- Convolutional outputs are flattened into vectors 


\section{Model Architecture}
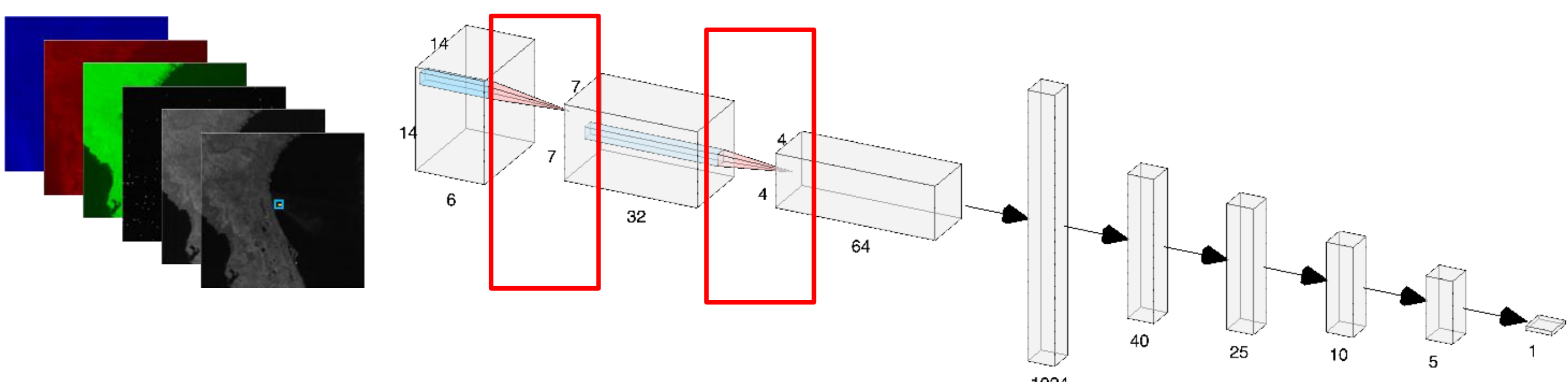

\begin{tabular}{|c|c|c|c|c|}
\hline 495 & 685 & 600 & 595 & $\ldots$ \\
\hline 515 & 665 & 595 & 550 & $\ldots$ \\
\hline 510 & 650 & 710 & 675 & $\ldots$ \\
\hline 490 & 705 & 675 & 625 & $\ldots$ \\
\hline 450 & 555 & 600 & 620 & $\ldots$ \\
\hline$\ldots$ & $\ldots$ & $\ldots$ & $\ldots$ & $\ldots$ \\
\hline
\end{tabular}

\begin{tabular}{|c|c|c|}
\hline 685 & 600 & $\ldots$ \\
\hline 705 & 710 & $\ldots$ \\
\hline$\ldots$ & $\ldots$ & $\ldots$ \\
\hline
\end{tabular}




\section{Model Architecture}
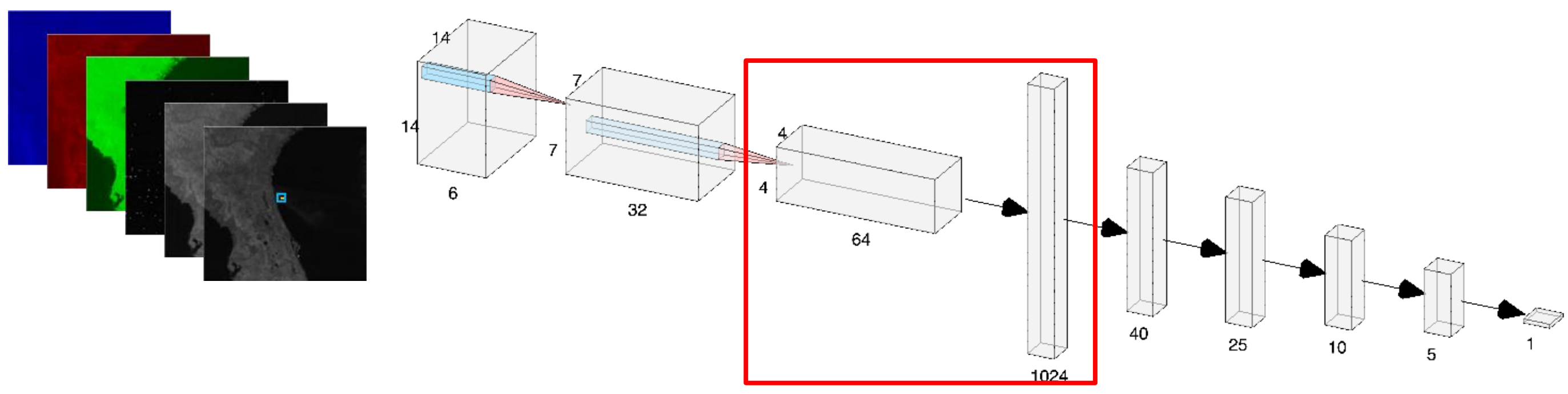

- Apply a pixel based Convolutional Neural Network (CNN)

- Input $\left(\mathrm{N}^{*} 2\right)^{*}\left(\mathrm{~N}^{*} 2\right)$ neighborhood of reflectance values surrounding a center pixel (sample)

- 3 convolutional layers

- Each followed by max-pooling layer

- Convolutional outputs are flattened into vectors 


\section{Model Architecture}
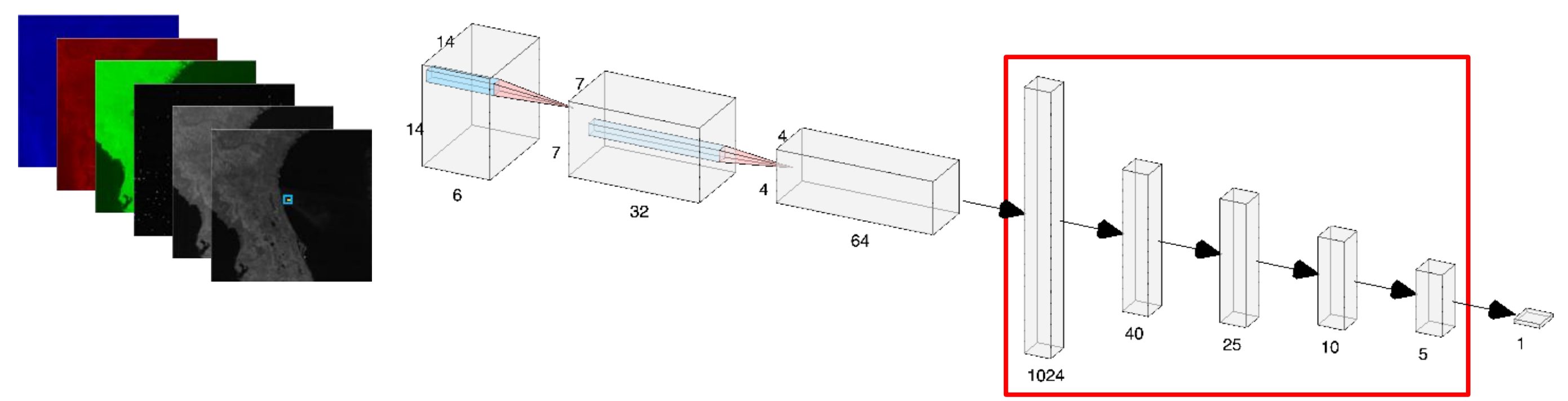

- Apply a pixel based Convolutional Neural Network (CNN)

- 4 fully connected layers with activation function calculation $g(W x+b)$

- $x$ is the flattened input vector

- $\mathrm{W}$ is the weight matrix

- $b$ is the bias vector

- Dropout for each fully connected layer 


\section{Model Architecture}
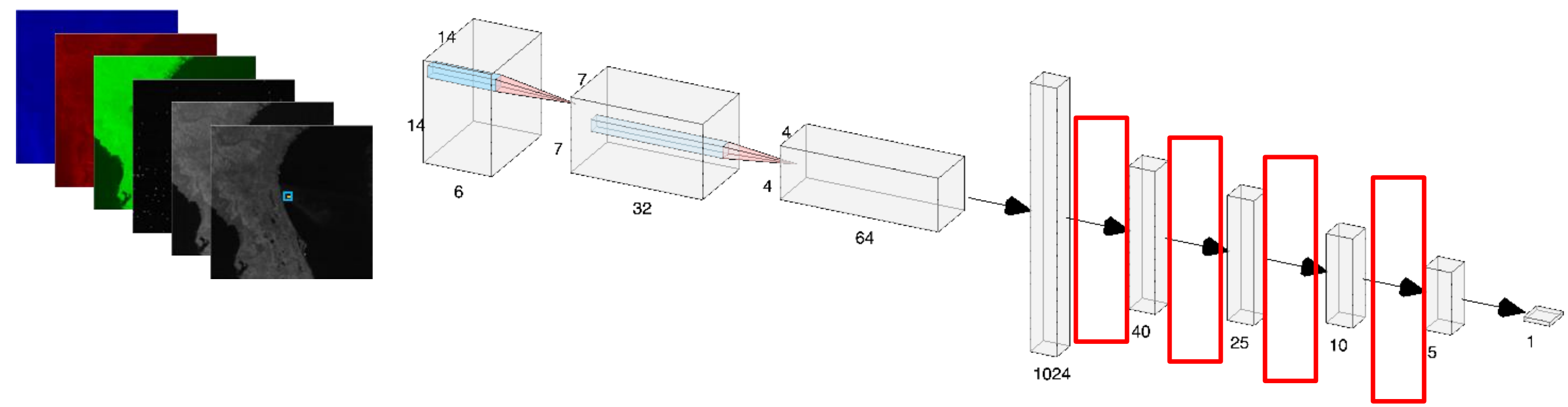

- Apply a pixel based Convolutional Neural Network (CNN)

- 4 fully connected layers with activation function calculation $g(W x+b)$

- $x$ is the flattened input vector

- $\mathrm{W}$ is the weight matrix

- $b$ is the bias vector

- Dropout randomly for each fully connected layer 


\section{Model Architecture}
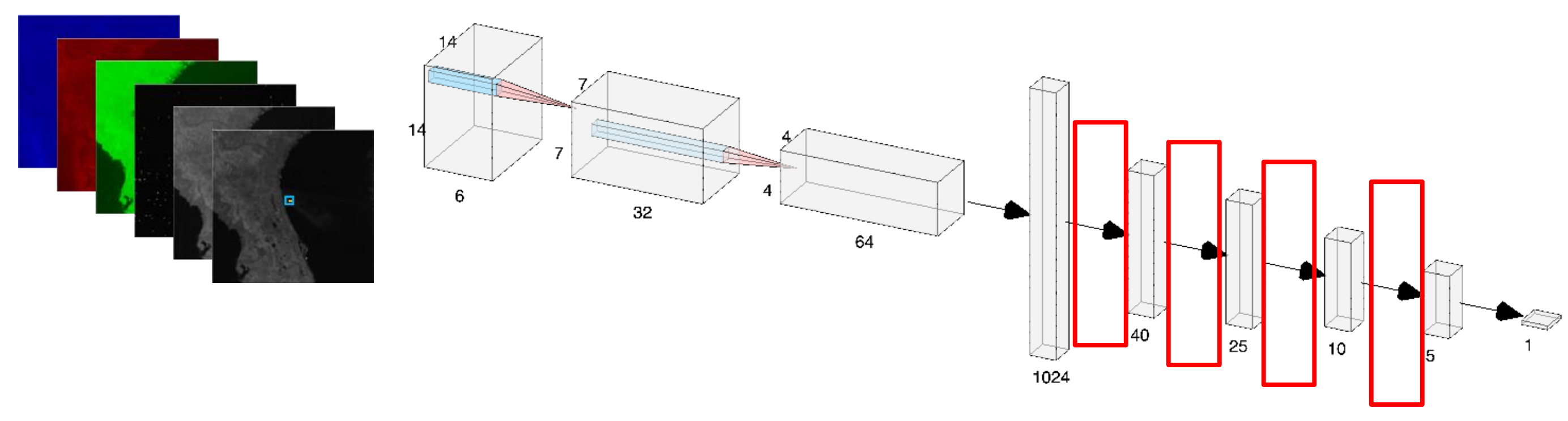

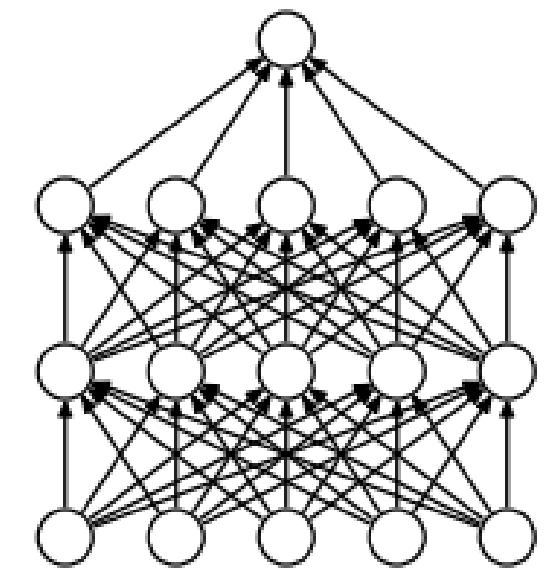

(a) Standard Neural Net

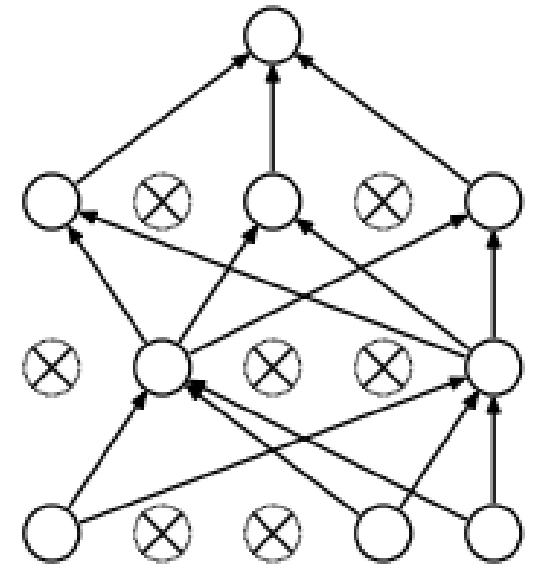

(b) After applying dropout. 


\section{Model Architecture}
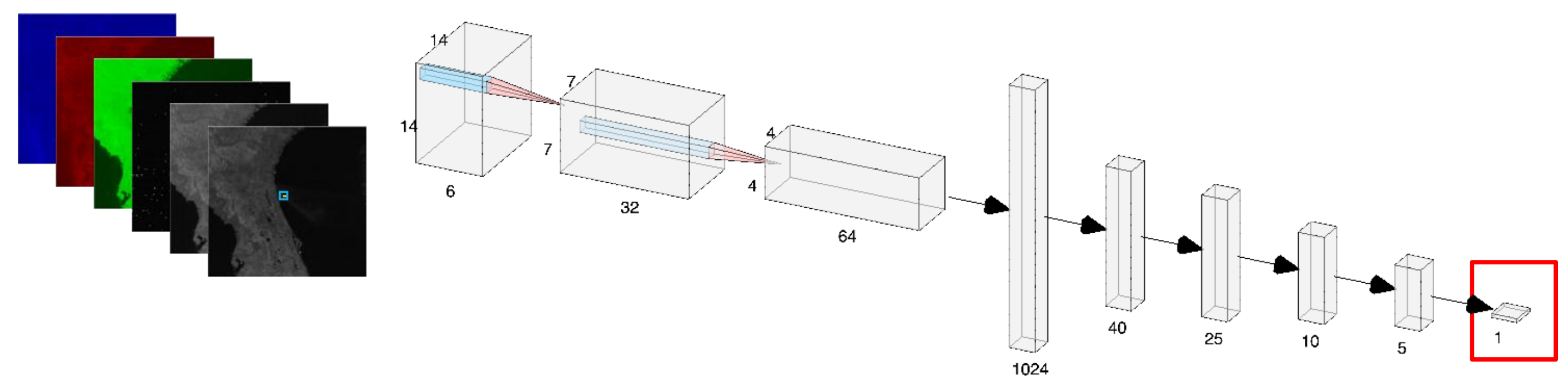

- The model outputs the probability, ranging from 0 to 1 , that a pixel is smoke determined by a sigmoid function

$$
p(x)=\frac{1}{1+e^{-x}}
$$

- $p>0.5$ threshold applied to define smoke 


\section{Neighborhood Selection}

- Best neighborhood size $(\mathrm{N})$ determined by iterating model development and testing for increasing $\mathrm{N}$

- All other parameters including data, learning rate and model hyper-parameters are held constant

- Best model selected when validation loss did not improve for 20 epochs

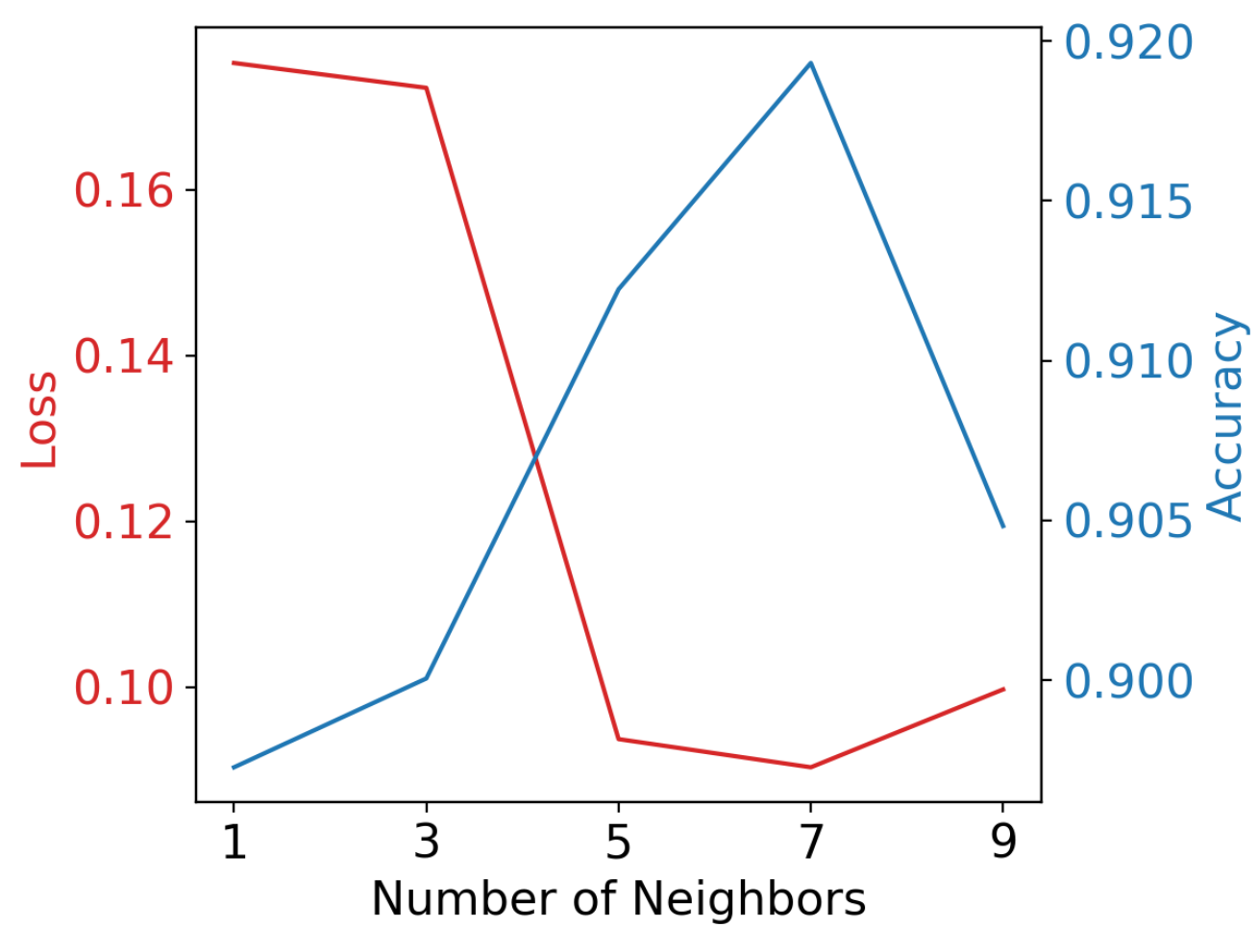




\section{Development Testing}

\begin{tabular}{|c|c|c|c|c|}
\hline $\mathrm{N}$ & Precision & Recall & F1-Score & Accuracy \\
\hline 1 & 0.654 & 0.328 & 0.437 & 0.897 \\
\hline 3 & 0.650 & 0.384 & 0.483 & 0.900 \\
\hline 5 & 0.724 & 0.449 & 0.554 & 0.912 \\
\hline 7 & 0.835 & 0.419 & 0.558 & 0.919 \\
\hline 9 & 0.639 & 0.498 & 0.560 & 0.905 \\
\hline
\end{tabular}

$$
\begin{gathered}
\text { Precision }=\frac{T P}{T P+F P} \\
\text { Recall }=\frac{T P}{T P+F N} \\
\text { Accuracy }=\frac{T P+T N}{T P+T N+T P+F N} \\
F 1 \text { Score }=2 * \frac{\text { Precision } * \text { Recall }}{\text { Precision }+ \text { Recall }}
\end{gathered}
$$

- The F1 Scores, or the harmonic mean of Precision and Recall, for $\mathrm{N}=5,7,9$ is comparable

- Trade-off between quality and quantity of smoke predictions

- Best model has low false positive detection rate which drives high precision

- Prefer conservative identification over incorrect classification

- Accuracy artifact of large number of True Negatives 


\section{Results}

- Model updated to account for variation in solar zenith angle

- The training and testing datasets for the updated model differ from that used for the initial development

- Results are comparable between the initial and updated models

- Better predictive capability of smoke over water

- Compared to land, the relative decrease in true negatives over water drives a slight decrease in accuracy

\begin{tabular}{|c|c|c|c|c|}
\hline $\mathrm{N}=7$ & Precision & Recall & F1-Score & Accuracy \\
\hline Dev. & 0.835 & 0.419 & 0.558 & 0.919 \\
\hline All & 0.736 & 0.453 & 0.561 & 0.923 \\
\hline Land & 0.631 & 0.383 & 0.476 & 0.928 \\
\hline Water & 0.923 & 0.585 & 0.717 & 0.900 \\
\hline
\end{tabular}




\section{May 2018 - Southern Florida}

- Smoke identified over both land and ocean

- Model identifies well defined plumes for scenes with absence of complex features

- Probabilities resemble visually observed optical thickness

- Predictions closer resemblance to quality controlled shapefiles

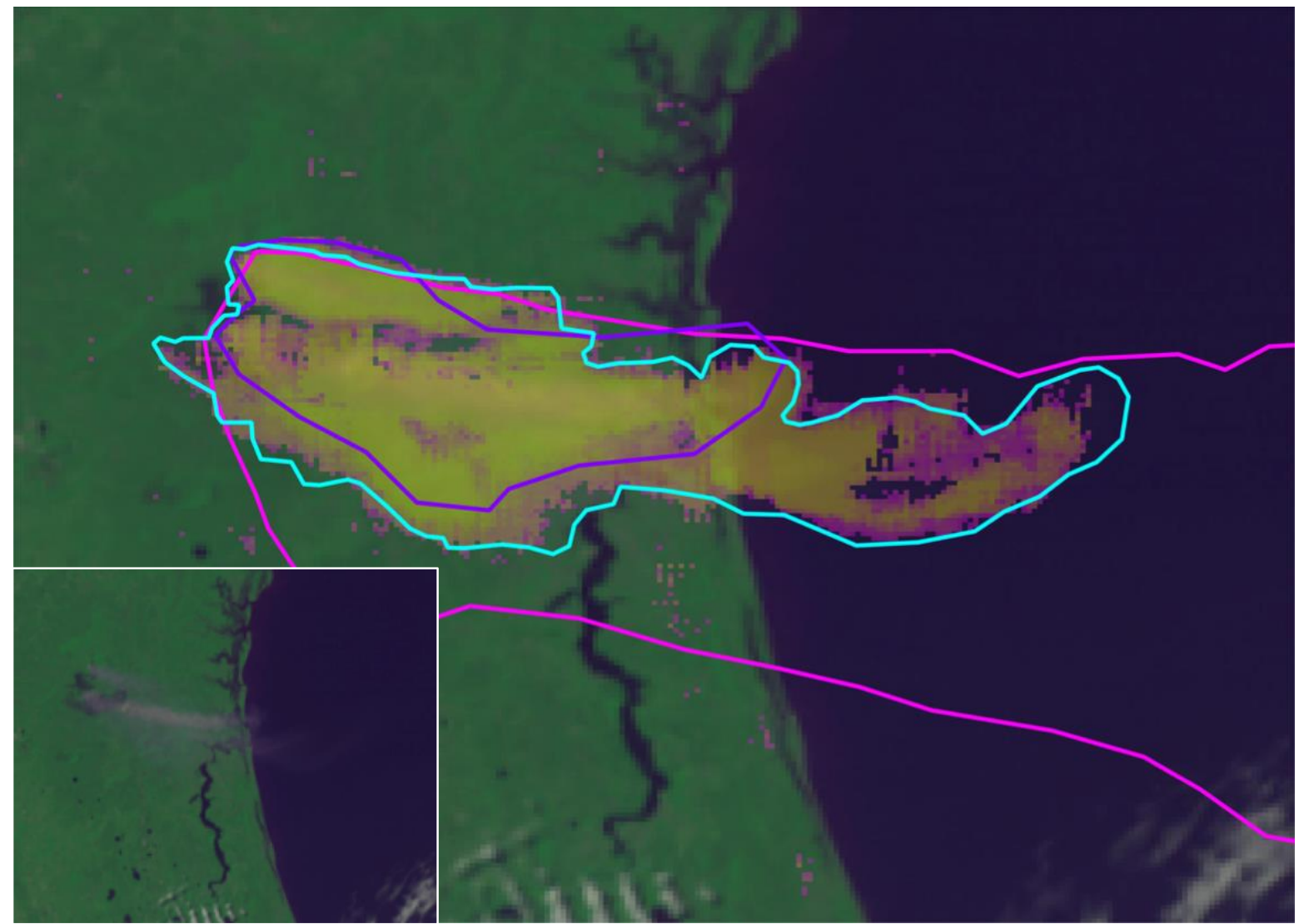

GOES 16 pseudo-RGB with contoured model predictions (shading), HMS shapefiles (magenta and purple), and subject matter quality controlled shapefile (blue). 


\section{March 2018 - Southern Florida}

- Distinguishable from chlorophyll commonly found in coastal settings

- Discriminate smoke from fair weather cumulus cloud

- Spectral information for other classes not provided to the model

\begin{tabular}{|c|c|c|c|c|}
\hline & Precision & Recall & F1-Score & Accuracy \\
\hline All & 0.744 & 0.604 & 0.666 & 0.948 \\
\hline Land & 0.847 & 0.244 & 0.379 & 0.976 \\
\hline Water & 0.742 & 0.623 & 0.677 & 0.943 \\
\hline
\end{tabular}

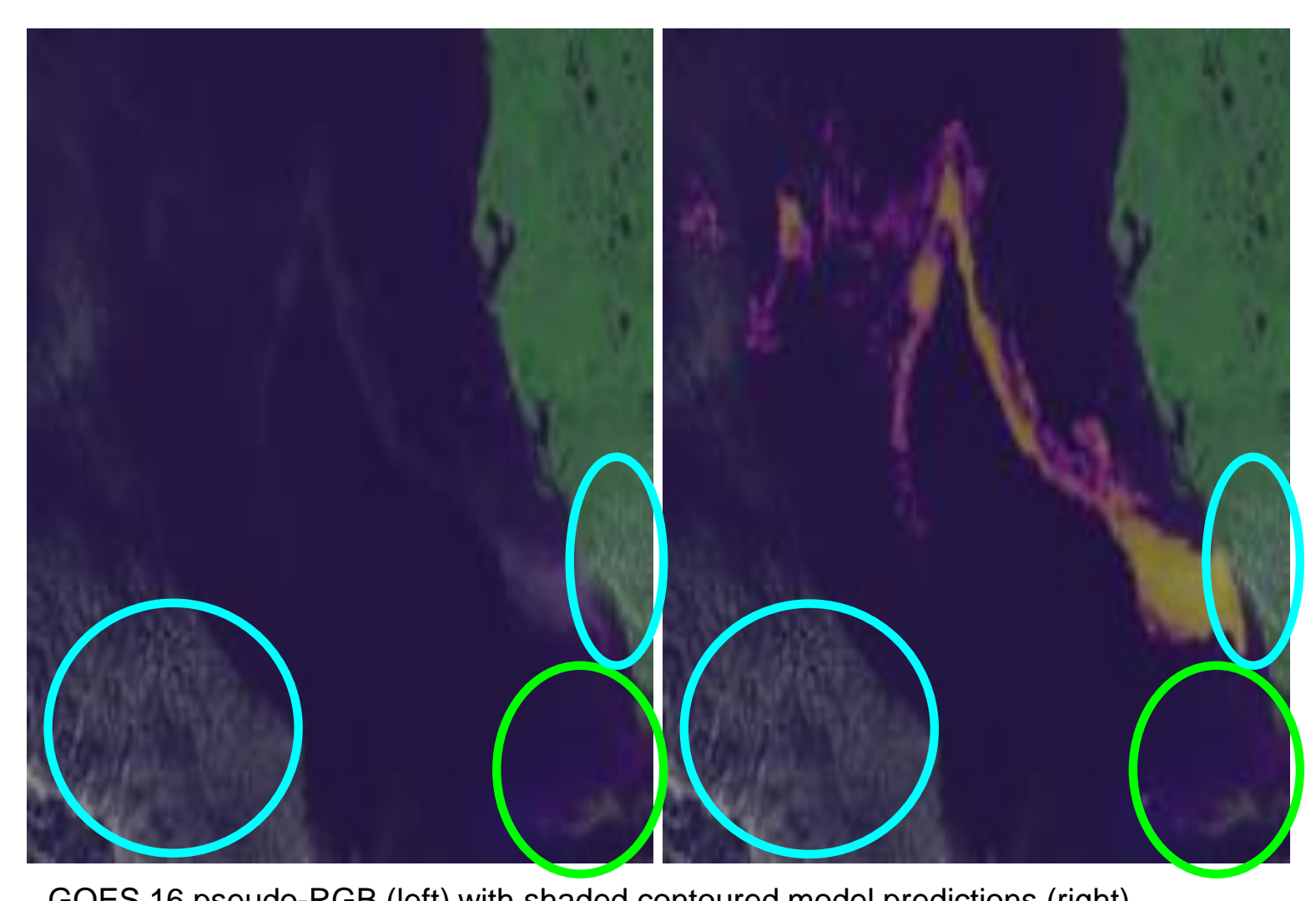




\section{June 2017 - Southern Rocky Mts. United States}

- Successfully discriminates land surface snow/ice from smoke

- Over snow capped mountains for this case

- Detection challenges for optically thin smoke over arid regions

\begin{tabular}{|c|c|c|c|c|}
\hline & Precision & Recall & F1-Score & Accuracy \\
\hline All & 0.848 & 0.318 & 0.462 & 0.977 \\
\hline Land & 0.848 & 0.319 & 0.463 & 0.977 \\
\hline Water & N/A & N/A & N/A & 0.984 \\
\hline
\end{tabular}

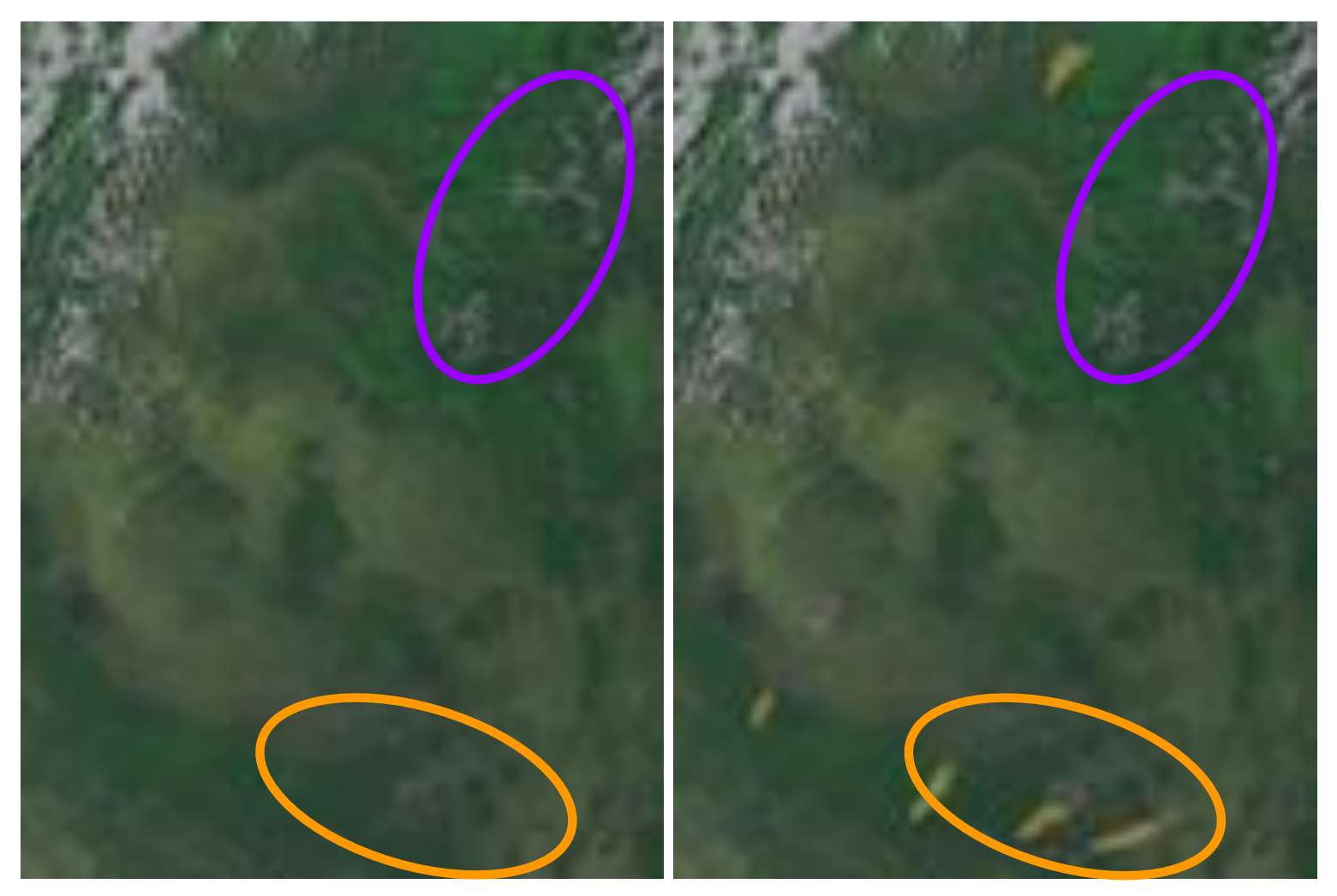

GOES 16 pseudo-RGB (left) with shaded contoured model predictions (right). 


\section{October 2017 - Central California}

- Large and small plumes

- Identification over both land and ocean

- Coastal stratus clouds

\begin{tabular}{|c|c|c|c|c|}
\hline & Precision & Recall & F1-Score & Accuracy \\
\hline All & 0.970 & 0.919 & 0.944 & 0.961 \\
\hline Land & 0.904 & 0.754 & 0.823 & 0.920 \\
\hline Water & 0.986 & 0.965 & 0.975 & 0.980 \\
\hline
\end{tabular}

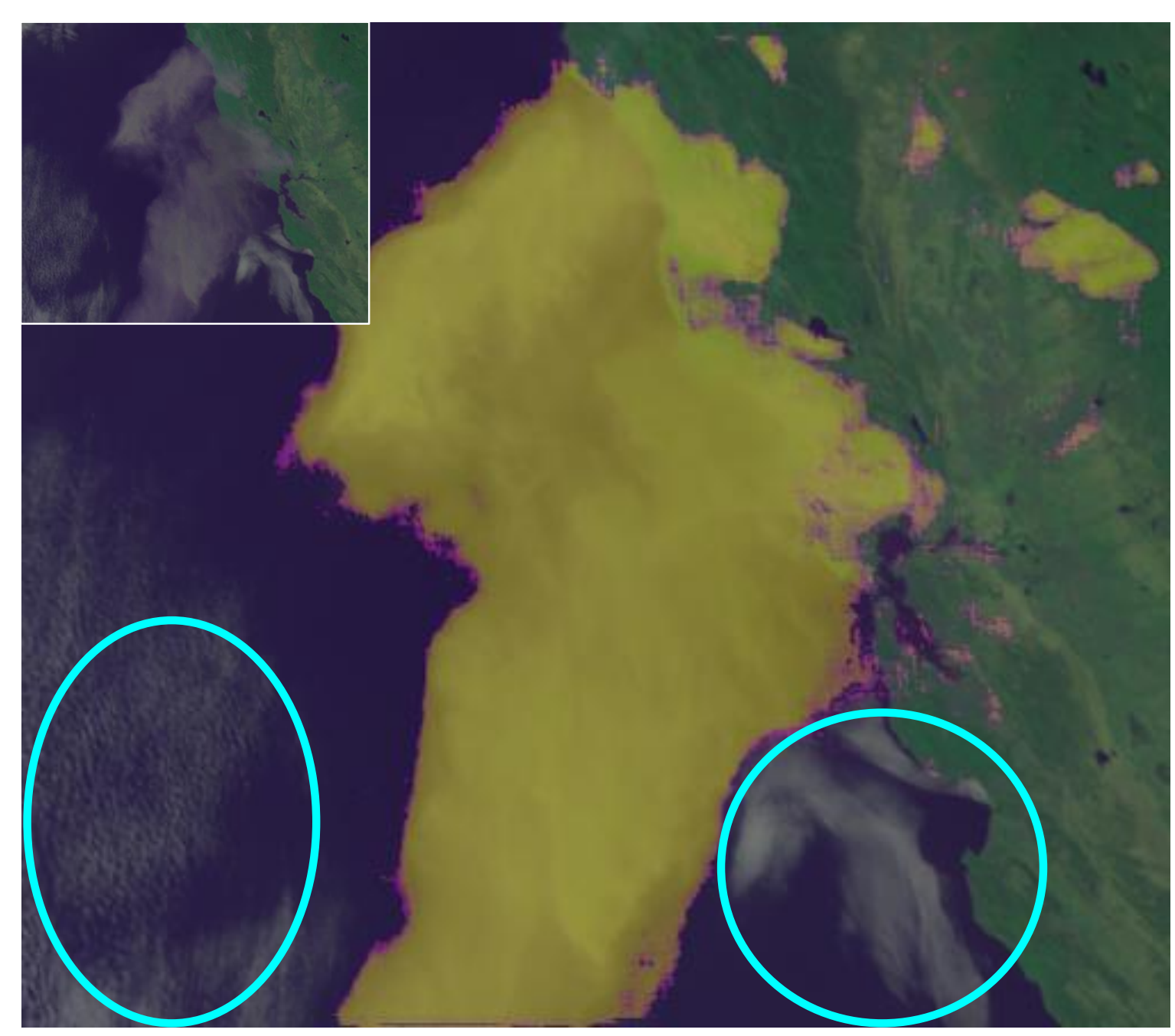




\section{May 2018 - Southern Arizona}

- Smoke not detected at very low sun angles

- Compounded by low optical thickness over relatively high reflective surface

- Probability of being smoke is low for few pixels that are identified

\begin{tabular}{|c|c|c|c|c|}
\hline & Precision & Recall & F1-Score & Accuracy \\
\hline All & 0.995 & 0.093 & 0.171 & 0.823 \\
\hline Land & 0.995 & 0.093 & 0.171 & 0.822 \\
\hline Water & 0.923 & 0.585 & 0.717 & 1.000 \\
\hline
\end{tabular}

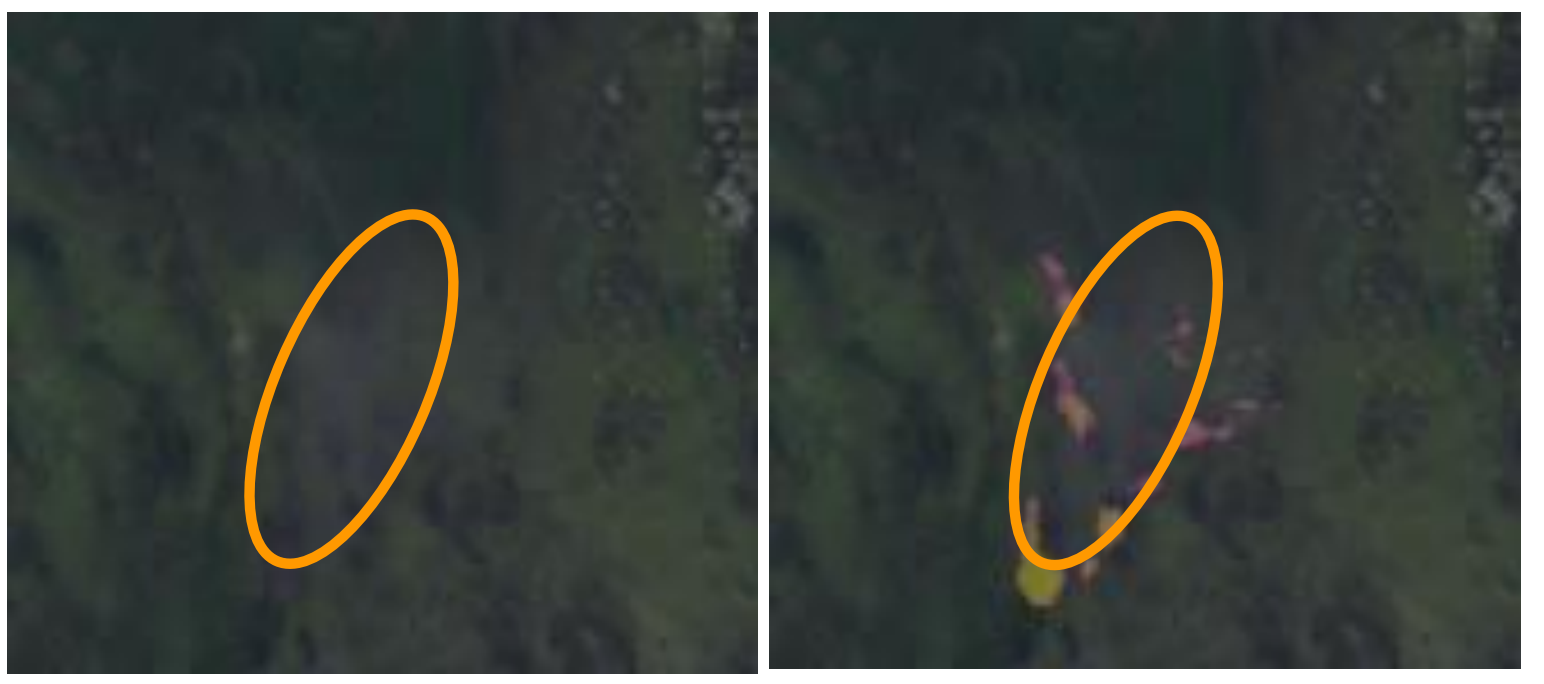

GOES 16 pseudo-RGB (left) with shaded contoured model predictions (right). 


\section{April 2018 - Southern Rocky Mts. United States}

- Overprediction of plume extent

- Artifact of large $(\mathrm{N}=7)$ neighborhood size

- Non-zero floor to number of false positives

\begin{tabular}{|c|c|c|c|c|}
\hline & Precision & Recall & F1-Score & Accuracy \\
\hline All & 0.830 & 0.738 & 0.781 & 0.981 \\
\hline Land & 0.830 & 0.738 & 0.781 & 0.981 \\
\hline Water & N/A & N/A & N/A & 0.993 \\
\hline
\end{tabular}

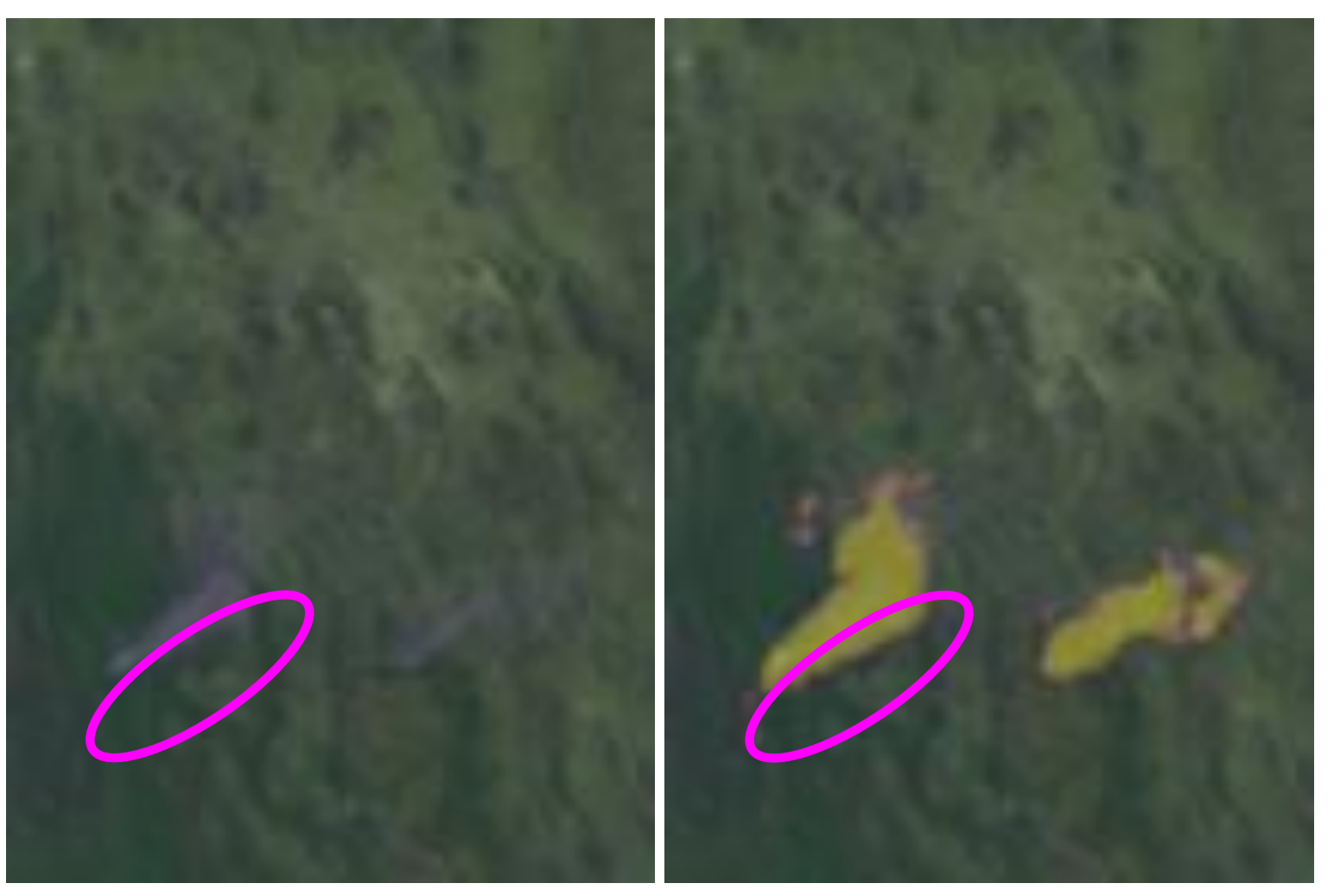




\section{April 2018 - Southern Rocky Mts. United States}

- Other atmospheric aerosols not classified as smoke

- Large dust storm case

- Represents a major source of aerosols in the atmosphere

- Expected over regions where smoke is also common

\begin{tabular}{|c|c|c|c|c|}
\hline & Precision & Recall & F1-Score & Accuracy \\
\hline All & N/A & N/A & N/A & 0.996 \\
\hline Land & N/A & N/A & N/A & 0.996 \\
\hline Water & N/A & N/A & N/A & N/A \\
\hline
\end{tabular}

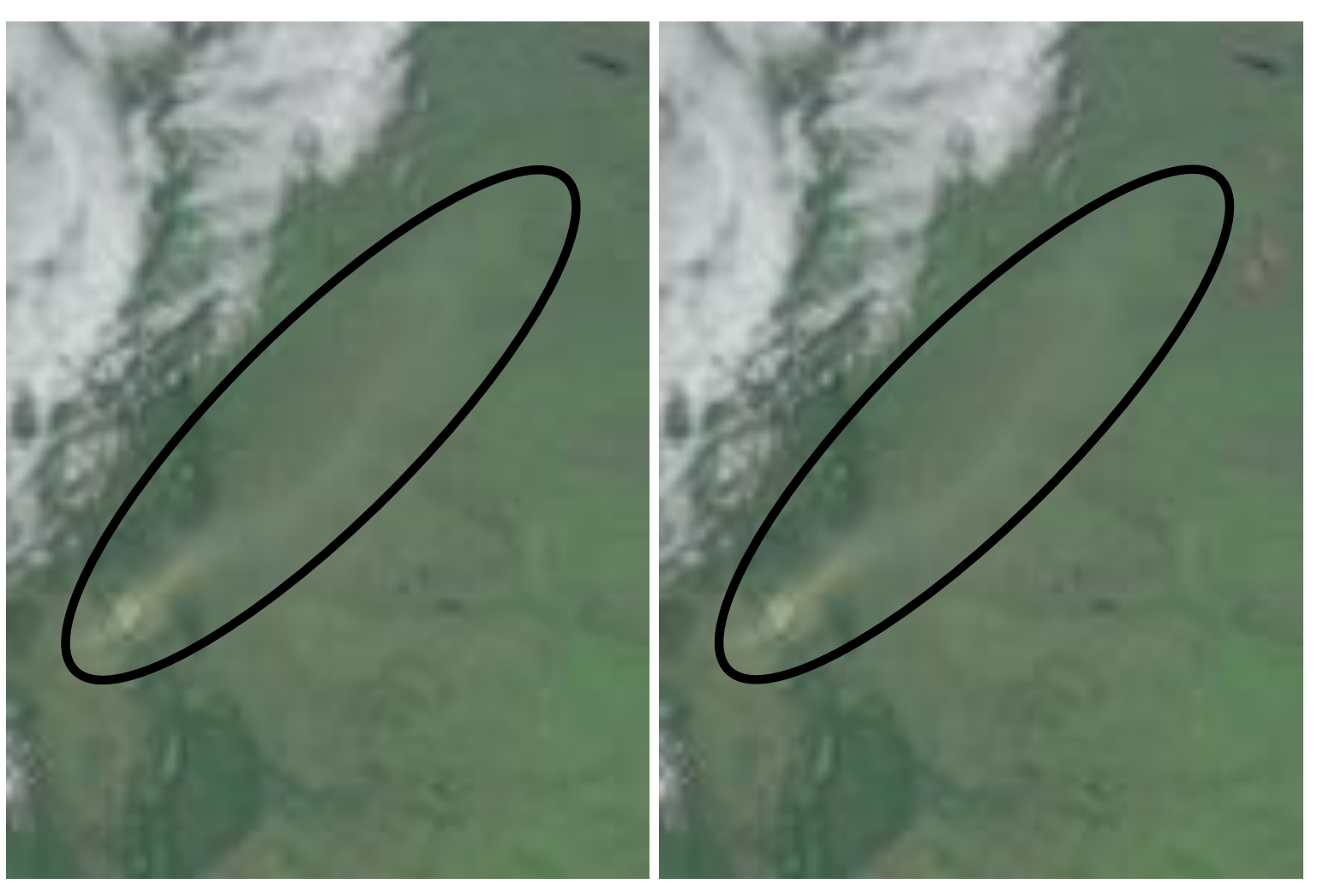

GOES 16 pseudo-RGB (left) with shaded contoured model predictions (right). 


\section{Operational Capabilities}

- Currently testing new deployment in operational environment - Anticipate operational October 2019

- Fully deployed in the cloud using Amazon S3 and Cloud Computing Services

- End-to-end prediction and visualization pipeline

- Model prediction available $\sim 15$ min after data availability

- Preprocessing 10 min

- Prediction and Postprocessing 5min

- Full disk GOES observation available $\sim 10$ min intervals 


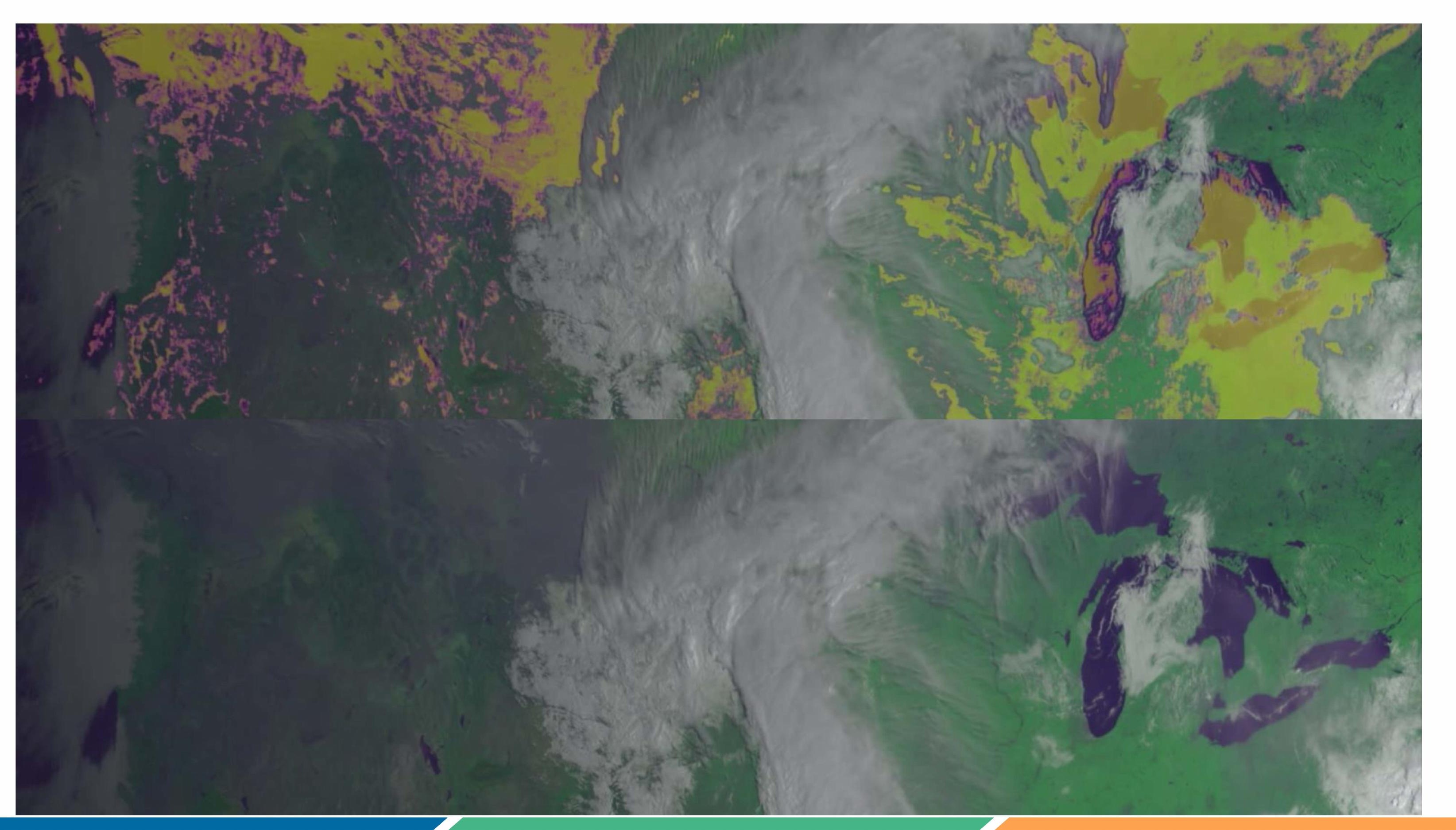




\section{Operational Capabilities - Postprocessing}

- Spatial grouping of predicted pixels to define plumes

- Convert predicted pixels to bitmap image

- Blurring to smoothen edges

- Contour blurred image to group smoke pixels into plumes

- Plumes visualized and geojson representation of plume extents available for download in the Phenomena Portal (http://phenomena.surge.sh)

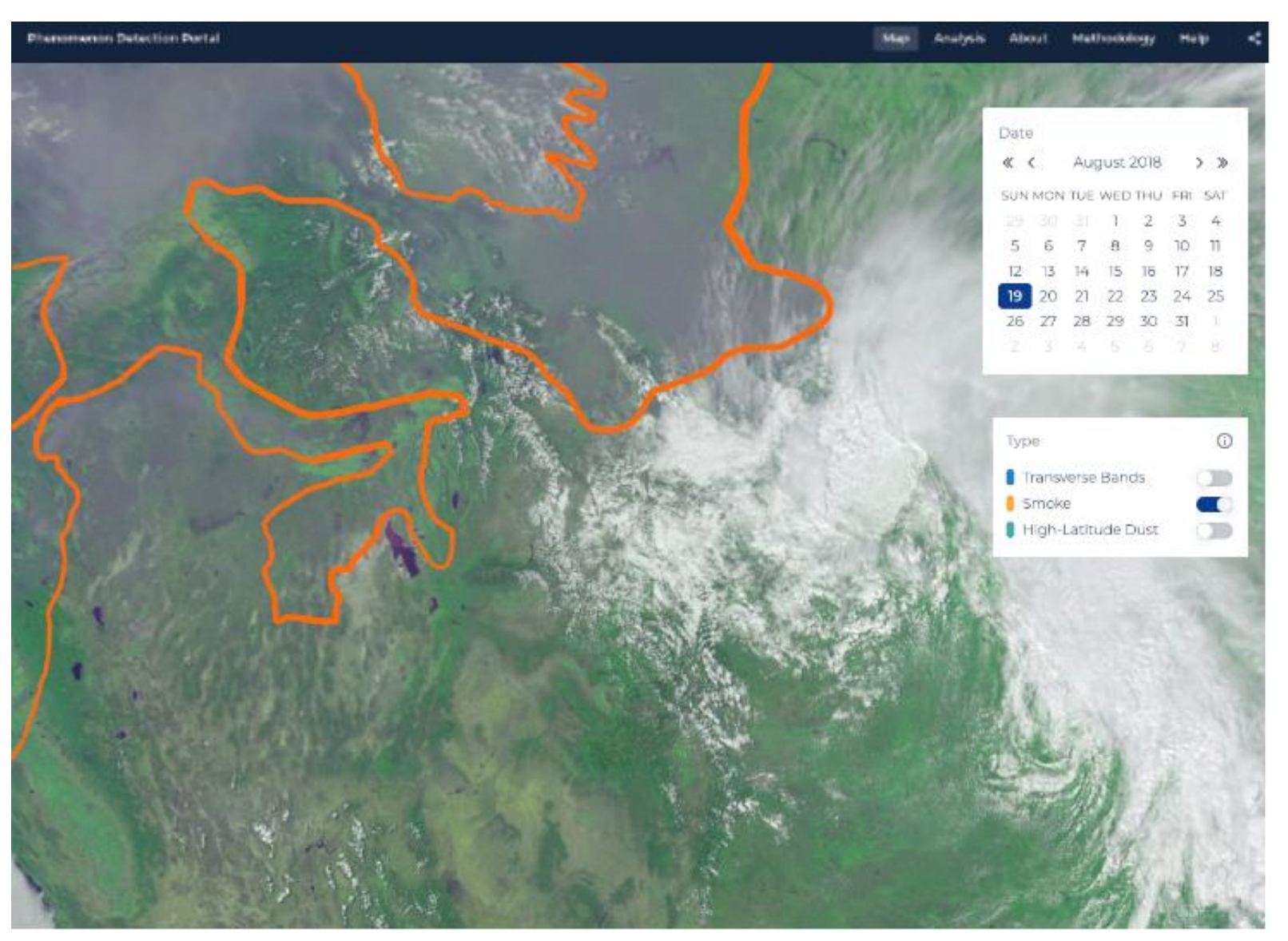




\section{Summary}

- Developed end-to-end machine learning smoke detection pipeline for next-generation of geostationary satellites

- Well curated smoke extent dataset

- Scalable smoke detection deep learning model, requiring only smoke information, and capable of detecting smoke with:

- Varying optical thicknesses

- Over low and high reflectance background surfaces

- Discriminates from features with spectral similarities

- Fully automated operational deployment of model in development

- Plume visualization and extent data accessible in online platform 


\section{Future work}

- Expand the training data to account for identified weaknesses

- Low sun angles

- Thin smoke over arid regions

- Thin clouds

- Refinement of the machine learning model

- Confirmation of $\mathrm{N}=7$ as best performing model

- Explore trade-off between neighborhood size and prediction capabilities

- Stepwise band selection considering all 16 GOES bands

- Robust model validation

- Band exclusion to identify contribution to feature learning

- Performance assessment for operational improvements 


\title{
Thank you!
}

\section{mr0051@uah.edu}

\author{
aaron.kaulfus@nsstc.uah.edu
}

NASA 